\title{
Temperature effects on the mechanical reliability of MEMS structures: experimental study on creep and thermal fatigue
}

\author{
G. De Pasquale, E. Brusa, and A. Somà \\ Mechanical Department, Politecnico di Torino, Corso Duca degli Abruzzi 24, 10129 Torino (Italy) \\ E-mail: giorgio.depasquale@polito.it, eugenio.brusa@polito.it, aurelio.soma@polito.it
}

\section{Introduction}

The reliability of micro electro-mechanical systems (MEMS) became a fundamental topic of investigation as a result of their widespread application in many day-to-day life devices. From the structural viewpoint the most relevant sources of lifetime reduction are mechanical fatigue and high temperatures. The typical working conditions of MEMS include repetitive cyclic loads and high operative frequencies; examples are represented by some of the most common applications: RF devices (switches, varactors, resonators, filters), inertial sensors, optical components, etc. These operative conditions activate that processes commonly associated to fatigue, like crack initiation and propagation and the self-heating of the material accompanied to the progressive degradation of mechanical properties. The fatigue associated to MEMS structures was investigated by the authors in some previous studies [1] providing experimental results in good agreement with the literature.

High temperatures are responsible of additional mechanisms of material degradation leading to a reduction of components lifetime and devices reliability. The most diffused scenarios associate high temperature to: a) static loads and b) alternate loads. In the first case creep effect appears: the temperature is responsible of progressively increasing deflections of the structure under the applied load; the deformation is usually plastic (and so, permanent) and large. In the second case the cause of material damaging is actually mechanical fatigue, but the presence of high levels of temperature modifies the S-N curve obtained at ambient temperature and generally determines faster failures at lower load levels.

This work is focused on the reliability of metallic MEMS structures subjected to static and alternate loads in presence of increasing levels of temperature. Some works in the literature were focused on the study of creep in MEMS that appears when static loads are associated to medium and high temperatures: Modlinski et al. [2] characterized the bridge of a capacitive switch made by an Al alloy, Larsen et al. [3] presented the design for a test structure devoted to investigation on creep for electroplated nickel, Zhang and Dunn [4] studied creep on thin gold-polysilicon bilayer films subjected to thermal loading. It was observed that long-term deformation of metal parts of microdevices may play a key role in the lifetime of a device, making creep a very important issue in the reliability of MEMS. Apart effects associated with diffusion, all plastic deformation occurs as a consequence of the migration of atomic planes in the crystal lattice of the material (namely dislocations); this effect is deeply emphasized by the coexistence of increasing temperature and stress. If alternate loads act on the component, the effect of temperature produces an acceleration of material damaging due to mechanical fatigue process; local plasticized area can be detected on the surface.

This is an Open Access article distributed under the terms of the Creative Commons Attribution-Noncommercial License 3.0, which permits unrestricted use, distribution, and reproduction in any noncommercial medium, provided the original work is properly cited. 


\section{Experiments}

The behavior of gold MEMS mechanical components in presence of increasing temperature is studied by using test structures designed ad-hoc and fabricated by the B. Kessler Foundation (FBK) located in Trento (Italy). The surface micromachining technique based on the gold electroplating process is used for the fabrication [5]. Simple structures like double clamped beams of variable dimensions (Figure 1a) are tested as well as more complex components like perforated plates suspended on elastic beams (Figure 1b). The first type of specimen reproduces the shape of RF resonators and filters; they can also be considered as the sub-component of more complex structures, like RF filters, varactors and proof mass of inertial sensors, the shape of which is reproduced by the second specimen. Each sample is subjected to static and alternate loads provided by an electrostatic actuation between the structure and the counter electrode situated on the ground. For static actuations, the sample deflection dependence to time and temperature is measured, providing a characterization of creep. For alternate loads, the number of cycles to failure is measured as a function of alternate stress and temperature, providing a characterization of mechanical fatigue as a function of temperature.

(a)

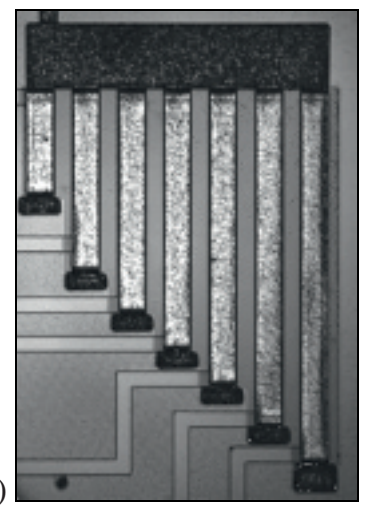

(b)

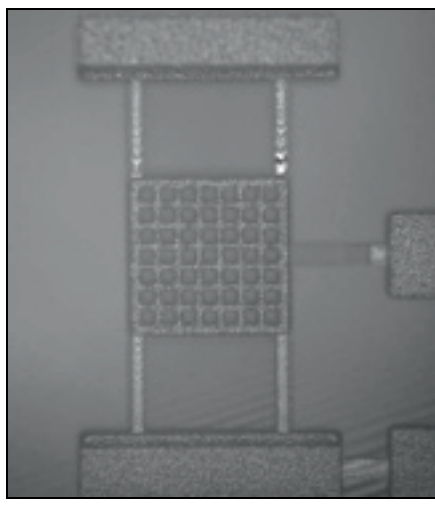

Fig. 1. Test structures analyzed: a) double clamped beams and b) perforated plates on elastic suspensions.

The measurements are performed by an interferometric microscope (ZoomSurf 3D, Fogale Nanotech). The microscope can perform profile measurements from a minimum area of $100 \times 100 \mathrm{~m}^{2}$ to a maximum of $2 \times 2 \mathrm{~mm}^{2}$. The actual setting provides a $20 \mathrm{x}$ objective magnification factor that reaches a $0.6 \mathrm{~m}$ and a $0.1 \mathrm{~nm}$ lateral and vertical resolutions, respectively. A single measurement can detect a $400 \mathrm{~m}$ maximum difference in height. The instrument is equipped with a 3 -axis movable piezo-electrical stage and a voltage generator $(0-200 \mathrm{~V}$, up to $2 \mathrm{MHz})$. The temperature variation is provided by a Peltier cell $\left(\mathrm{T}_{\max }=120^{\circ} \mathrm{C}\right)$, controlled by a PT100 temperature sensor.

\section{References}

1. A. Somà, G. De Pasquale, J. Microelectromech. S. 18, 828-835 (2009)

2. R. Modlinski, A. Witvrouw, P. Ratchev, A. Jourdain, V. Simons, H.A.C. Tilmans, J.M.J. den Toonder, R. Puers, I. De Wolf, Microelectron. Reliab. 44, 1733-1738 (2004)

3. K.P. Larsen, A.A. Rasmussen, J.T. Ravnkilde, M. Ginnerup, O. Hansen, Sensors Actuat. APhys. 103, 156-164 (2003)

4. Y. Zhang, M.L. Dunn, J. Mech. Phys. Solids 52, 2101-2126 (2004)

5. B. Margesin, A. Bagolini, I. Guarnieri, F. Giacomozzi, A. Faes, proc. DTIP, 402-405 (2003) 